\title{
ON THE SCHWARZIAN COEIFICIENTS OF THE KOEBE TRANSFORM OF A UNIVALENT FUNCTION
}

\author{
STEPHEN M. ZEMYAN
}

\begin{abstract}
For $f \in S$, we compute the Schwarzian coefficients of the Koebe Transform $f_{\phi_{\alpha}}$ of $f$ in terms of successive derivatives of the Schwar- zian derivative, then provide some estimates on certain combinations of them.
\end{abstract}

Let $S$ denote the class of functions $f(z)=z+a_{2} z^{2}+\ldots$ which are analytic and univalent in the unit disk $U=\{z:|z|<1\}$.

The Schwarzian derivative of a function $f(z)$ in $S$ is defined by the relation

$$
\{f, z\}=\left(\frac{f^{\prime \prime}(z)}{f^{\prime}(z)}\right)^{\prime}-\frac{1}{2}\left(\frac{f^{\prime \prime}(z)}{f^{\prime}(z)}\right)^{2}
$$

and the Schwarzian coefficients of the function $f(z)$ are the Taylor coefficients in the series expansion

$$
\{f, z\}=\sum_{n=0}^{\infty} s_{n} z^{n}
$$

In a previous paper, the author [14] determined sharp estimates on the coefficients $s_{0}, s_{1}$ and $s_{2}$, as well as a general estimate on $s_{n}$, and general estimates on certain linear combinations of Schwarzian coefficients. In this paper, we determine explicit formulas for the Schwarzian coefficients of the Koebe Transform of a function $f \in S$ in terms of the successive derivatives of the Schwarzian derivative, and then provide growth estimates on certain combinations of them.

Received December 26, 1991. 
Let $w(z)$ be an analytic, univalent function in $U$ with $|w(z)|<1$. If $f \in S$, then

$$
f_{w}(z)=\frac{f(w(z))-f(w(0))}{f^{\prime}(w(0)) w^{\prime}(0)}
$$

also belongs to $S$. The Schwarzian derivatives of $f$ and $f_{w}$, and hence their coefficients, are related by the composition law $[4$, p.376]

$$
\left\{f_{w}, z\right\}=\{f, w(z)\}\left(w^{\prime}(z)\right)^{2}+\{w, z\} .
$$

If we now choose

$$
w(z)=\phi_{\alpha}(z)=\frac{z-\alpha}{1-\bar{\alpha} z}=-\alpha+\left(1-|\alpha|^{2}\right) \sum_{m=1}^{\infty} \bar{\alpha}^{m-1} z^{m},(\alpha \in U)
$$

then $f_{w}=f_{\phi_{a}} \in S$ is known as the Koebe Transform of $f$, and the composition law becomes

$$
\left\{f_{\phi_{\alpha}}, z\right\}=\left\{f, \phi_{\alpha}(z)\right\}\left(\phi_{\alpha}^{\prime}(z)\right)^{2} .
$$

Let us investigate the Schwarzian coefficients of $f_{\phi_{\alpha}}$. If we write, for $n \geq 0$,

$$
\left(\phi_{\alpha}(z)\right)^{n}=\sum_{m=0}^{\infty} c_{m}^{(n)}(\alpha) z^{m}
$$

then

$$
\begin{aligned}
\left\{f, \phi_{\alpha}(z)\right\} & =\sum_{n=0}^{\infty} s_{n}(f)\left(\phi_{\alpha}(z)\right)^{n} \\
& =\sum_{m=0}^{\infty} C_{m}(f ; \alpha) z^{m}
\end{aligned}
$$

where

and

$$
C_{0}(f ; \alpha)=\sum_{n=0}^{\infty} s_{n}(f) c_{0}^{(n)}(\alpha)
$$

Since

$$
C_{m}(f ; \alpha)=\sum_{n=1}^{\infty} s_{n}(f) c_{m}^{(n)}(\alpha) . \quad(m \geq 1)
$$

$$
\left(\phi_{\alpha}^{\prime}(z)\right)^{2}=\left(1-|\alpha|^{2}\right)^{2} \sum_{m=0}^{\infty}\left(\begin{array}{c}
m+3 \\
3
\end{array}\right) \bar{\alpha}^{m} z^{m}
$$


a comparison of coefficients in the composition law shows that the Schwarzian coefficients of the Koebe Transform are given by

$$
s_{n}\left(f_{\phi_{\alpha}}\right)=\left(1-|\alpha|^{2}\right)^{2} \sum_{m=0}^{n}\left(\begin{array}{c}
n+3-m \\
3
\end{array}\right) \bar{\alpha}^{n-m} C_{m}(f ; \alpha) .
$$

In order to estimate these coefficients and linear combinations of them, we must first determine the sums $C_{m}(f ; \alpha)(m=0,1,2, \ldots)$.

Lemma. Let $f \in S$ and let $\{f, z\}^{(r)}(r=1,2,3, \ldots)$ denote the rth derivative of $\{f, z\}$. Then,

$$
C_{0}(f ; \alpha)=\{f,-\alpha\}
$$

and, for $m \geq 1$,

$$
C_{m}(f ; \alpha)=\sum_{r=1}^{m} \frac{1}{r !}\left(\begin{array}{c}
m-1 \\
r-1
\end{array}\right)\left(1-|\alpha|^{2}\right)^{r} \bar{\alpha}^{m-r}\{f,-\alpha\}^{(r)} .
$$

Proof. We must first determine the coefficients $c_{m}^{(n)}(\alpha)$ defined by (5). It is clear from (4) that $c_{0}^{(0)}(\alpha)=1$, given by $c_{m}^{(0)}(\alpha)=0(m=1,2, \ldots)$, and that $c_{0}^{(n)}(\alpha)=(-\alpha)^{n}(n=1,2,3, \ldots)$. Clearly, $C_{0}(f ; \alpha)=\sum_{n=0}^{\infty} s_{n}(f)(-\alpha)^{n}=$ $\{f,-\alpha\}$. For all $n, m \geq 1$, a tedious induction on $n$, using the relation given by $c_{m}^{(n+1)}(\alpha)=\sum_{j=0}^{m} c_{j}^{(n)}(\alpha) \cdot c_{m-j}^{(1)}(\alpha)$, shows that

$$
c_{m}^{(n)}(\alpha)=\sum_{r=1}^{m}\left(\begin{array}{c}
m-1 \\
r-1
\end{array}\right)\left(\begin{array}{l}
n \\
r
\end{array}\right)\left(1-|\alpha|^{2}\right)^{r} \bar{\alpha}^{m-r}(-\alpha)^{n-r},
$$

where it is understood that $\left(\begin{array}{l}n \\ r\end{array}\right)=0$, if $r>n$. Substituting (9) into (6) and carefully interchanging sums establishes (8) for all $m \geq 1$.

In [14], the author established the sharp inequalities $\left|s_{0}\right| \leq 6,\left|s_{1}\right| \leq 16$ and $\left|s_{2}\right| \leq 30\left(1+2 e^{-34 / 3}\right)$. As of a consequence of these estimates and the above lemma, we may obtain sharp bounds on the quantities

$$
\begin{aligned}
& s_{0}\left(f_{\phi_{\alpha}}\right)=\left(1-|\alpha|^{2}\right)^{2}\{f,-\alpha\} \\
& s_{1}\left(f_{\phi_{\alpha}}\right)=\left(1-|\alpha|^{2}\right)^{2}\left[4 \bar{\alpha}\{f,-\alpha\}+\left(1-|\alpha|^{2}\right)\{f,-\alpha\}^{(1)}\right]
\end{aligned}
$$

and 


$$
\begin{aligned}
s_{2}\left(f_{\phi_{\alpha}}\right)=(1 & \left.-|\alpha|^{2}\right)^{2}\left[10 \bar{\alpha}^{2}\{f,-\alpha\}+5 \bar{\alpha}\left(1-|\alpha|^{2}\right)\{f,-\alpha\}^{(1)}\right. \\
& \left.+\frac{1}{2}\left(1-|\alpha|^{2}\right)^{2}\{f,-\alpha\}^{(2)}\right]
\end{aligned}
$$

for any $\alpha \in U$. Also, an application of the triangle inequality now shows that

$$
\left|\{f, z\}^{(1)}\right| \leq \frac{16+24|z|}{\left(1-|z|^{2}\right)^{3}}
$$

which is sharp if $z=0$, and has the correct order of growth for $|z|$ near 1 . This inequality may be used in turn to provide an estimate for $\left|\{f, z\}^{(2)}\right|$.

We are now ready to state our general result. Here, $(a)_{r}$ represents the Appell symbol defined by $(a)_{0}=1$ and $(a)_{r}=a(a+1) \ldots(a+r-1)$ for $r \geq 1$.

Theorem. Let $f \in S$ and let $f_{\phi_{\alpha}}$ denote the Koebe Transform of $f$. Then, the Schwarzian coefficients of $f_{\phi_{\alpha}}$ are given by

$$
s_{n}\left(f_{\phi_{\alpha}}\right)=\left(1-|\alpha|^{2}\right)^{2}\left(\begin{array}{c}
n+3 \\
3
\end{array}\right) \sum_{r=0}^{n} \frac{(-1)^{r}(-n)_{r}}{(4)_{r}(1)_{r}}\left(1-|\alpha|^{2}\right)^{r} \bar{\alpha}^{n-r}\{f,-\alpha\}^{(r)}
$$

for all $n \geq 0$, where $\{f,-\alpha\}^{(r)}(r=0,1,2, \ldots)$ denotes the rth derivative of $\{f, z\}$ evaluated at $z=-\alpha$. Consequently, for every $N \geq 0$, we have

where

$$
\left|\sum_{r=0}^{N} \frac{1}{(4)_{r}(1)_{r}} P(N, r ; \bar{\alpha})\left(1-|\alpha|^{2}\right)^{r}\{f,-\alpha\}^{(r)}\right| \leq \frac{(N+1)_{3}}{\left(1-|\alpha|^{2}\right)^{2}}
$$

$$
P(N, r ; \bar{\alpha})={ }_{2} F_{1}(-N+r, r+4 ;-N+r-1 ; \bar{\alpha}) .
$$

is a hypergeometric polynomial of degree $(N-r)$ in the variable $\bar{\alpha}$.

Proof. Substituting (8) into (7), and then interchanging sums, we obtain

$$
\begin{aligned}
\frac{s_{n}\left(f_{\phi_{\alpha}}\right)}{\left(1-|\alpha|^{2}\right)^{2}}= & \left(\begin{array}{c}
n+3 \\
3
\end{array}\right) \bar{\alpha}^{n}\{f,-\alpha\} \\
& +\sum_{r=1}^{n}\left[\sum_{m=r}^{n}\left(\begin{array}{c}
n+3-m \\
3
\end{array}\right)\left(\begin{array}{c}
m-1 \\
r-1
\end{array}\right)\right] \frac{1}{r !}\left(1-|\alpha|^{2}\right)^{r} \bar{\alpha}^{n-r}\{f,-\alpha\}^{(r)} \\
= & \left(\begin{array}{c}
n+3 \\
3
\end{array}\right) \bar{\alpha}^{n}\{f,-\alpha\} \\
& +\sum_{r=1}^{n}\left(\begin{array}{c}
n+3 \\
3
\end{array}\right) \frac{(-1)^{r}(-n)_{r}}{(4)_{r}(1)_{r}}\left(1-|\alpha|^{2}\right)^{r} \bar{\alpha}^{n-r}\{f,-\alpha\}^{(r)}
\end{aligned}
$$


which establishes (10). (The inner sum was evaluated by using Vandermonde's formula, after a shift of index.) Hence, after interchanging sums, we get

$\sum_{n=0}^{N}(N+1-n) s_{n}\left(f_{\phi_{\alpha}}\right)=\left(1-|\alpha|^{2}\right)^{2} \sum_{r=0} \frac{1}{(4)_{r}(1)_{r}} P(N, r ; \bar{\alpha})\left(1-|\alpha|^{2}\right)^{r}\{f,-\alpha\}^{(r)}$

where

$$
\begin{aligned}
P(N, r ; \bar{\alpha}) & =\sum_{n=r}^{N}(N+1-r)\left(\begin{array}{c}
n+3 \\
3
\end{array}\right)(-1)^{r}(-n)_{r} \bar{\alpha}^{n-r} \\
& =\sum_{n=0}^{N-r}(N+1-n-r)\left(\begin{array}{c}
n+r+3 \\
3
\end{array}\right)(-n-r)_{r} \bar{\alpha}^{n} \\
& =\sum_{n=0}^{N-r} p_{n} \bar{\alpha}^{n}
\end{aligned}
$$

Since

$$
\frac{p_{n+1}}{p_{n}}=\frac{(-N+r+n)(r+4+n)}{(-N+r-1+n)(1+n)}
$$

it is clear that

$$
p_{n}=\frac{(-N+r)_{n}(r+4)_{n}}{(-N+r-1)_{n}} \cdot \frac{1}{n !}
$$

so that $P(N, r ; \bar{\alpha})$ is a hypergeometric polynomial of degree $(N-r)$ in $\bar{\alpha}$, as we have claimed. The conclusion (11) now follows directly from the inequality

$$
\left|\sum_{n=0}^{N}(N+1-n) s_{n}\right| \leq(N+1)(N+2)(N+3)
$$

which was established in [14, Theorem 3].

\section{References}

[1] P. L. Duren, "Univalent Functions", Springer-Verlag, Heidelberg and New York, 1983.

[2] G. M. Goluzin, "Geometric Theory of Functions of a Complex Variable", English Transl., Amer. Math. Soc., Providence, R. I., 1969.

[3] R. Harmelin, "Generalized Grunsky coefficients and inequalities", Israel J. of Math., Vol. 57, No. 3. (1987), 347-364.

[4] E. Hille, "Analytic Function Theory", II, Chelsea, New York, 1962.

[5] J. A. Hummel, "The Grunsky coefficients of a schlicht function", Proc. Amer. Math. Soc., 15 (1964), 142-150. 
[6] J. A. Jenkins, "On certain coefficients of univalent functions" in Analytic Functions, Princeton Univ. Press, Princeton, N. J., 1960.

[7] W. Kraus, "Über den Zusammenhang einiger Charakteristiken eines einfach zusammenhängenden Bereiches mit der Kreisabbildung", Mitt. Math. Sem. Giessen, 21 (1932), 1-28.

[8] Ch. Pommerenke, "Univalent Functions", Vanderhoeck and Ruprecht, Göttingen, 1975.

[9] M. Schiffer, "Sur un problème d'extemum de la représentation conforme", Bulb. Soc. Math. France, 66 (1938), 48-55.

[10] G. Schober, "Univalent Functions-Selected Topics", Lecture Notes in Math. No. 478, Springer-Verlag, 1975.

[11] I. Schur, "On Faber Polynomials", Amer. J. Math., 67 (1945), 33-41.

[12] P. Todorov, "Explicit Formulas for the coefficients of Faber polynomials with respect to univalent functions of the class $\sum$ ", Proc. Amer. Math. Soc., Vol. 82, Number 3, (1981), 431-438.

[13] C. Fitzgerald and Ch. Pommerenke, "The de Branges theorem on univalent functions", Trans. Amer. Math. Soc., 290 (1985), 683-690.

[14] S. Zemyan, "On the Schwarzian Coefficients of Univalent Functions", to appear. in the Bulletin of the Australian Mathematical Society, 46 (1992),389-398.

Department of Mathematics, The Pennsylvania State University, Mont Alto Campus, Mont Alto, Pennsylvania 17237-9799, U. S. A. 